\title{
Improved MRSI With Field Inhomogeneity Compensation
}

\author{
Ildar Khalidov ${ }^{a}$, Dimitri Van De Ville ${ }^{a}$, Mathews $\operatorname{Jacob}^{b}$, François Lazeyras $^{c}$, Michael Unser $^{a}$ \\ ${ }^{a}$ Biomedical Imaging Group, EPFL, Lausanne, Switzerland; \\ ${ }^{b}$ Beckman Institute, University of Illinois at Urbana-Champaign, Urbana, IL, USA; \\ ${ }^{c}$ Department of Radiology and Medical Informatics, HUG, Geneva, Switzerland
}

\begin{abstract}
Magnetic resonance spectroscopy imaging (MRSI) is a promising and developing tool in medical imaging. Because of various difficulties imposed by the imperfections of the scanner and the reconstruction algorithms, its applicability in clinical practice is rather limited. In this paper, we suggest an extension of the constrained reconstruction technique (SLIM). Our algorithm, named B-SLIM, takes into account the the measured field inhomogeneity map, which contains both the scanner's main field inhomogeneity and the object-dependent magnetic susceptibility effects. The method is implemented and tested both with synthetic and physical two-compartment phantom data. The results demonstrate significant performance improvement over the SLIM technique. At the same time, the algorithm has the same computational complexity as SLIM.
\end{abstract}

\section{INTRODUCTION}

Nonspectroscopic magnetic resonance imaging (MRI) is widely used in current clinical practice due to its ability to create high-resolution images of the interior body in a non-invasive way. In MRI, spatial locations of the voxels are encoded by the frequencies in the MR signal. The scanner provides a set of so-called $k$-space samples from this signal, on which reconstruction (typically, the Fourier transform) should be performed to obtain the final image. Although MRI allows to image a large variety of contrasts (e.g., proton density, $T_{1}, T_{2}$ ), it does not acquire the full spectral information as in traditional magnetic resonance spectroscopy (MRS). The combination of the resolution and localization of MRI with the spectroscopic richness of MRS has a high potential for many clinical applications.

For this purpose, magnetic resonance spectroscopy imaging (MRSI) has been proposed. Basically, it measures the chemical shift at several $k$-space positions. This procedure requires phase-encoding; so, the acquisition time per $k$-space point is long. Clearly, keeping the experiment duration reasonable makes the attainable spatial resolution for MRSI much lower than for MRI, typically, $16 \times 16$. This low resolution and the large concentration differences of some metabolites between adjacent tissues exacerbate the artifacts of Fourier series reconstruction. ${ }^{1,2}$ Among other solutions, ${ }^{3-5}$ the constrained reconstruction method ${ }^{6,7}$ (SLIM) exploits additional information of spatially homogeneous compartments that is extracted from high-resolution MR images. The SLIM method allows to efficiently recover the compartmental spectra; e.g., as shown for the human limb. However, particularly in brain imaging, the influence of the main scanner field $B_{0}$ inhomogeneity and the magnetic susceptibility effects near the tissue boundaries are substantial. Their combined effect, even after having carefully applied a shimming technique, can shift the local spectrum by several units of the ppm-scale. Therefore, the hypothesis that the compartments are spatially homogeneous is no longer valid and the method is unable to adequately resolve the spectra.

In this paper, we propose an extension to the SLIM algorithm that includes a suitable compensation for the $B_{0}$-inhomogeneity effects, named B-SLIM. As in SLIM, we start from a high-resolution MRI scan of the object, e.g., a proton density image, which is then segmented into compartments that are considered as spectroscopically uniform. Additionally, we use an AUTOSHIM-like technique ${ }^{8}$ to measure the $B_{0}$ inhomogeneity map, which includes the scanner field inhomogeneity and the object-dependent magnetic susceptibility effects. Both the

Further author information:

(ildar.khalidov, dimitri.vandeville, michael.unser)@epfl.ch

mjacob@uiuc.edu

francois.lazeyras@sim.hcuge.ch

Medical Imaging 2006: Image Processing, edited by Joseph M. Reinhardt, Josien P. W. Pluim, Proc. of SPIE Vol. 6144, 614467, (2006) · 0277-786X/06/\$15 · doi: 10.1117/12.651868 
information about the compartments and the inhomogeneity map are then fed as a priori information into our extended signal model. Finally, the spectra of the compartments are found as the solution of a least-squares formulation; i.e., we minimize the error between the signal model and the MRSI measurements. This solution can be found in a direct way by taking advantage of the block-diagonal structure of the formulation, leading to the same computational complexity as the SLIM algorithm.

The proposed method was implemented to demonstrate its feasibility. First, we generated a synthetic MRSI dataset; i.e., a virtual two-compartment phantom. This allowed us to verify the implementation and to show the theoretical effect of the field inhomogeneity on the spectra as found by SLIM. Next, we acquired data for a physical two-compartment phantom where the compartments contained $\mathrm{NAA}+\mathrm{Cr}$ in doped water and corn oil, respectively. When using SLIM, the fat peak from the corn oil contaminates the spectrum of the other compartment. Here, we show that B-SLIM is able to suppress these artifacts and recover the original expected spectra.

This paper is organized as follows. In Section 2, we give a brief overview of the theory that lies behind our method; in Section 3, we formulate the algorithm. Then, in Section 4, we describe the test setup that was used to probe the feasibility of the algorithm and illustrate the results including a comparison with SLIM. We then conclude with a discussion of possible applications of the algorithm.

\section{THEORY}

The proposed signal model in MRSI is

$$
p_{i}(t)=s\left(\mathbf{k}_{i}, t\right)=\int_{-\infty}^{\infty} \int_{\text {space }} \rho(\mathbf{x}, f) e^{-j \Delta \omega(\mathbf{x}) t-j 2 \pi\left(\mathbf{k}_{i} \cdot \mathbf{x}+f t\right)} \mathrm{d} \mathbf{x} \mathrm{d} f
$$

where

- $\rho(\mathbf{x}, f)$ is the spatio-spectral distribution that we are trying to reconstruct;

- $p_{i}(t)$ are the measurements obtained from an MRSI experiment;

- $\Delta \omega(\mathbf{x})=\gamma \Delta B_{0}(\mathbf{x})$, where $\Delta B_{0}(\mathbf{x})$ represents the inhomogeneity function and $B_{0}+\Delta B_{0}(\mathbf{x})$ is the magnitude of the apparent B0 field;

- $\gamma$ is the gyromagnetic ratio;

- the $\mathbf{k}_{i}$ 's define the k-space low-resolution measurement grid.

We use the SLIM model for the object; i.e., we assume that the object consists of $K$ spectroscopically uniform compartments, characterized by their spatial indicator functions $\chi_{k}(\mathbf{x})$ and their spectra $q_{k}(f), k=1, \ldots, K$. The overall density $\rho(\mathbf{x}, f)$ then takes the form

$$
\rho(\mathbf{x}, f)=\sum_{k=1}^{K} \chi_{k}(\mathbf{x}) q_{k}(f)
$$

For the measurements, we then have

$$
p_{i}(t)=s\left(\mathbf{k}_{i}, t\right)=\sum_{k=1}^{K} Q_{k}(t) \int_{\text {space }} \chi_{k}(\mathbf{x}) e^{-j \Delta \omega(\mathbf{x}) t-j 2 \pi \mathbf{k}_{i} \cdot \mathbf{x}} \mathrm{d} \mathbf{x}=: \sum_{k=1}^{K} Q_{k}(t) H_{k, i}(t),
$$

where $Q_{k}(t)$ is the Fourier transform of $q_{k}(f)$. We can now state our inverse problem as a least-square (LS) minimization: given the measurements $\left\{p_{i}(t)\right\}_{i=1, \ldots, M}$, the compartments $\chi_{k}(\mathbf{x})$ and the inhomogeneity map $\Delta \omega(\mathbf{x})$, find $Q_{k}(t)$ such that

$$
\sum_{i=1}^{M}\left\|p_{i}(t)-\sum_{k=1}^{K} H_{k, i}(t) Q_{k}(t)\right\|_{L_{2}}^{2} \rightarrow \min .
$$


In the general GSLIM framework, the number of unknowns is much higher than the number of equations, so the above criterion needs a regularization term. In B-SLIM, we add a priori constraints related to the measured B0 inhomogeneity distribution, and regularization is not needed anymore.

Interestingly, the standard SLIM case corresponds to choosing $\Delta \omega(\mathbf{x})=0$ (perfectly homogeneous field) in (3). If this is not the case, $H_{k, i}$, by definition (3), depends on $t$. However, if there are no constraints on $Q_{k}(t)$, we can reformulate the problem as a pointwise minimization in $t$; i.e., for each $t_{0}$, we look for $Q_{k}\left(t_{0}\right)$ such that

$$
\sum_{i=1}^{M}\left|p_{i}\left(t_{0}\right)-\sum_{k=1}^{K} H_{k, i}\left(t_{0}\right) Q_{k}\left(t_{0}\right)\right|^{2} \rightarrow \min .
$$

Thanks to this new formulation, we have the following highly efficient numerical algorithm.

\section{ALGORITHM}

1. Acquire the high-resolution proton density image and segment it into $K$ compartment regions $\chi_{k}(\mathbf{x})$, using either automatic or assisted segmentation techniques. Acquire or estimate the inhomogeneity map $\Delta B_{0}(\mathbf{x})$. Get the MRSI measurements $\left\{p_{i}(t)\right\}$, where the index $i$ corresponds to the $i$-th point of the $\mathrm{k}$-space measurement grid, and $t$ runs along the temporal dimension.

2. For each compartment, form the "template" structures $t_{k}(\mathbf{x}, f)$ : for every sample coordinate $\left(\mathbf{x}_{0}\right)$, the function $t_{k}\left(\mathbf{x}_{0}, f\right)$ approximates an impulse located at the frequency $\frac{1}{2 \pi} \Delta \omega\left(\mathbf{x}_{0}\right)$. Perform spatial FFTs to obtain the corresponding k-space structures, downsample them at the low-resolution measurement grid. Perform an FFT on the spectral dimension.

3. For each time point $t_{0}$, compute the LS solution

$$
Q_{k}\left(t_{0}\right)=\arg \min _{Q_{k}} \sum_{i}\left|p_{i}\left(t_{0}\right)-\sum_{k=1}^{K} H_{k, i}\left(t_{0}\right) Q_{k}\right|^{2}
$$

corresponding to (4). From the computational point of view, this LS solution boils down to a simple $K \times K$ matrix inversion; i.e., the algorithm is as efficient as classical SLIM.

\section{RESULTS}

The method was implemented in MATLAB. The core algorithm takes $K$ high-resolution compartment images, the B0 inhomogeneity map and MRSI measurements of size $16 \times 16 \times 1024$ as input, and computes the spectra for each compartment.

To demonstrate the feasibility of our method, we first show experiments using synthetic data. The object was modeled as a disc containing a hypothetical substance of which the spectrum has a single peak surrounded by a ring with another substance. To simulate magnetic susceptibility effects in the real scanner, a highpass-filtered version of the high-resolution inner-compartment image was taken as the B0 inhomogeneity map (see Fig. 1).

Secondly, the algorithm was tested with a physical phantom, which consisted of an "inner" bottle with a solution of NAA and Cr in doped water, and an "outer" bottle with corn oil.

All measurements were taken with a Philips Gyroscan 1.5T scanner. B0 inhomogeneity was measured after shimming by the means of an AUTOSHIM-like method. ${ }^{8}$ This method is fast (only two high-resolution MRI acquisitions are needed), and takes into account the effects introduced both by shimming and by the magnetic susceptibility differences. Next, the high-resolution proton image was taken and segmented using a simple watershed-based algorithm in MATLAB to determine the compartments (see Fig. 2). In our case, the number of compartments $K$ was equal to 2: the first compartment was the outer bottle (fat) and the second one was the inner bottle (metabolite solution).

For the synthetic data, the B-SLIM algorithm produced the results shown in Figures 3(a)-3(b). The corresponding result obtained with standard SLIM (Fig. 3(c)) shows the contamination of the adjacent compartment 


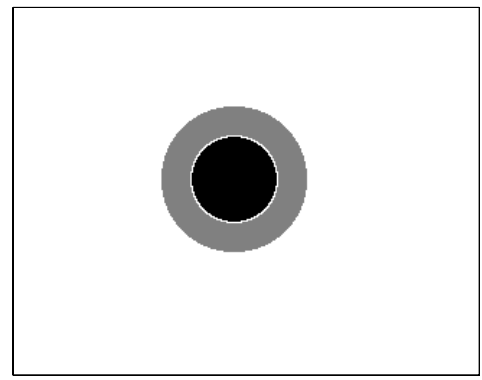

(a) Simulated object

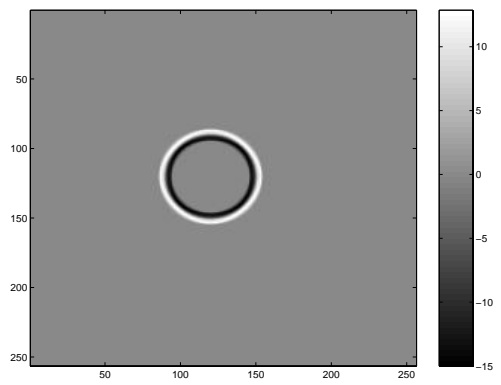

(d) Simulated B0 inhomogeneity map

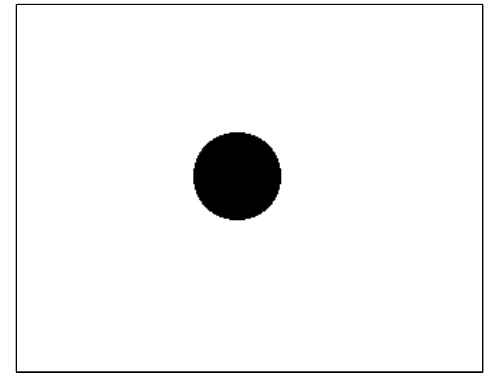

(b) Compartment 1: Indicator function

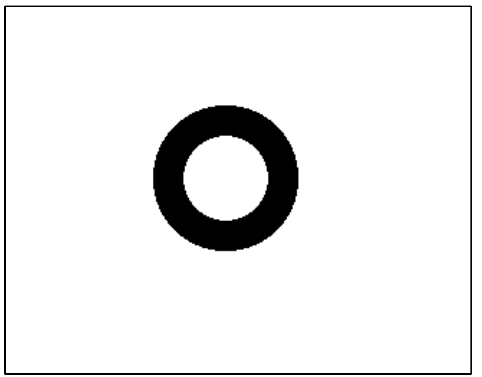

(e) Compartment 2: Indicator function

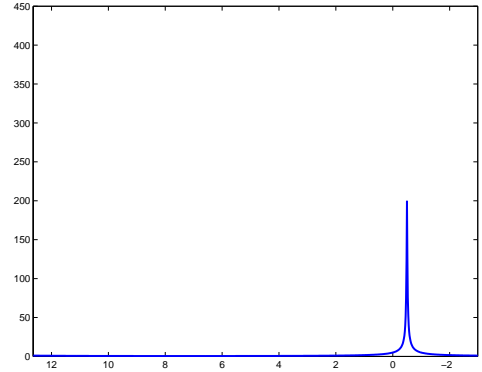

(c) Compartment 1: Synthetic spectrum

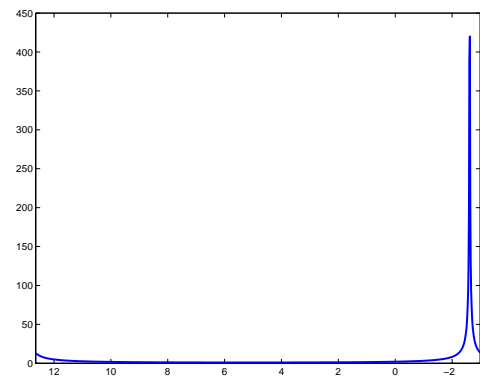

(f) Compartment 2: Synthetic spectrum

Figure 1. Synthetic data: The two-compartment object, the field inhomogeneity map, compartment indicator functions and the corresponding spectra

by the stronger peak. In this simulation, the propagated peak intensity is only two times higher; however, we can observe a visible false peak in the SLIM solution for the inner compartment, the height of which is about $1 / 3$ of that of the true peak.

The results of B-SLIM for the phantom experiment are shown in Figure 4. We provide the obtained spectra for the two compartments, together with a magnified version of the regions of interest. To understand the improvements that we get when comparing to the standard SLIM method, we also compute the spectrum for compartment 2 without inhomogeneity compensation. In Figure 4, we observe that the spurious peaks for the standard SLIM case are even higher than the metabolite peaks. The proposed algorithm finds spectra where the "real" peaks are much stronger. This is not only quantitative, but also an important qualitative result, as it allows clear differentiation between the true and spurious peaks. In addition, by comparing the absolute intensity of the peaks in Fig. 4, we observe an improvement in the sensitivity of the algorithm to the metabolite signal. More detailed experimental results will be included in our forthcoming paper. ${ }^{9}$

With the mentioned data volumes, the spectra reconstruction operation takes a few seconds on a Macintosh G5 with 1GB RAM.

\section{CONCLUSIONS}

We have proposed a new reconstruction method (B-SLIM) that solves the inverse problem in MRSI. Based on the ideas of SLIM, our method uses the MRI image of the object under investigation. At the same time, it takes into account the a priori information on the B0 field inhomogeneity. The suggested implementation is computationally efficient and comparable in execution speed with the MRSI-version of SLIM. 


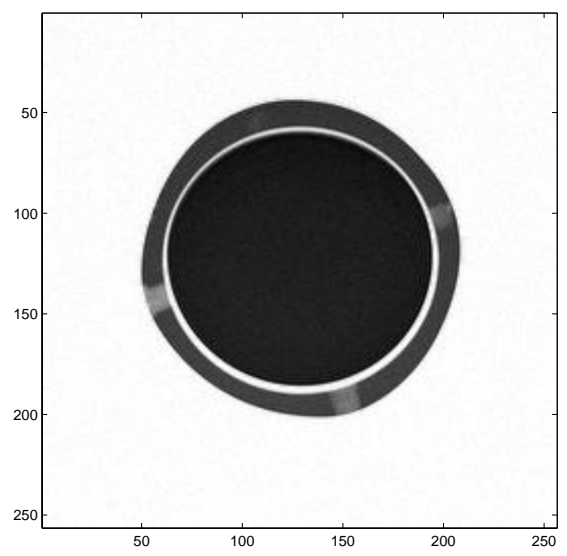

(a) High-resolution MRI image of the phantom

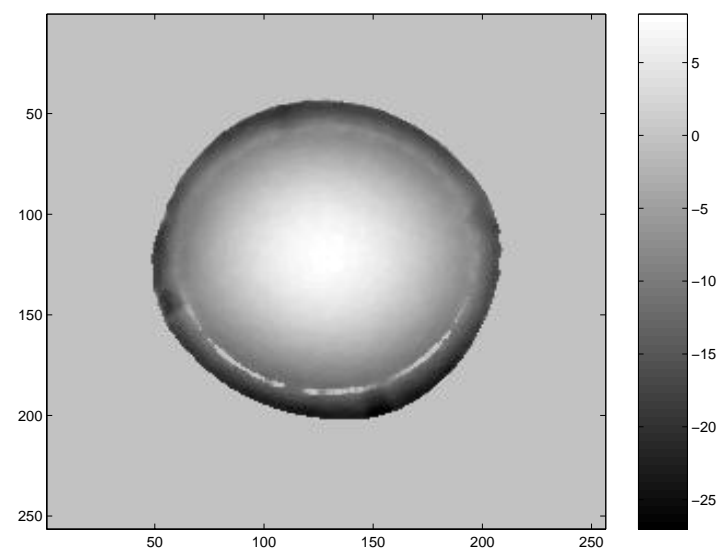

(b) Measured B0 inhomogeneity map

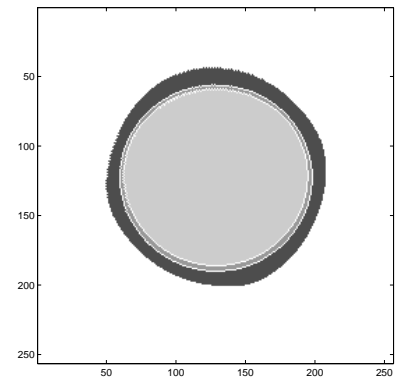

(c) Segmentation of the MRI image

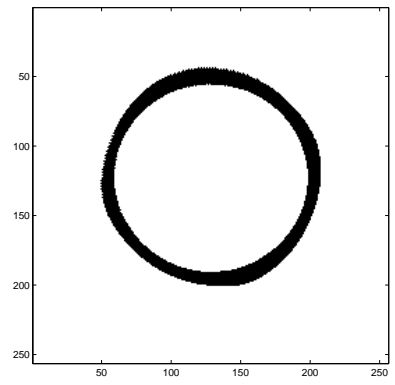

(d) Compartment 1: Indicator function

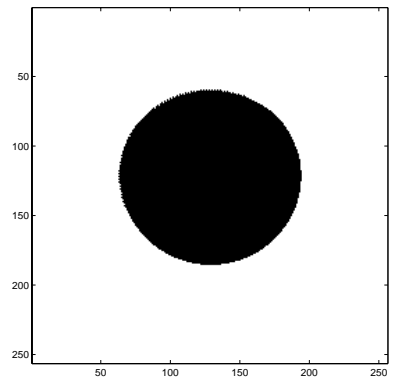

(e) Compartment 2: Indicator function

Figure 2. Phantom experiment: The MRI image of the phantom, the field inhomogeneity map and the compartment indicator functions

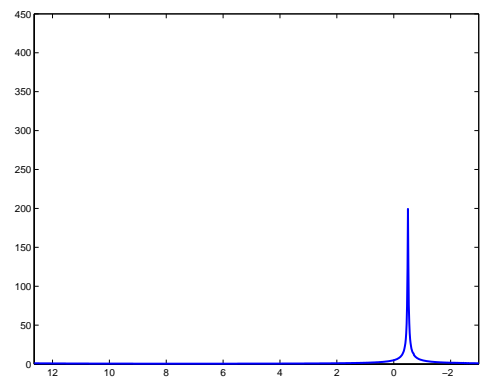

(a) Compartment 1: B-SLIMresolved spectrum

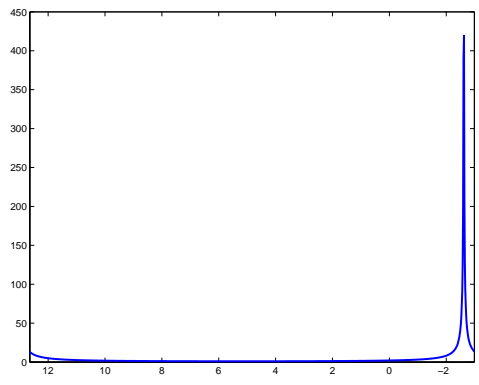

(b) Compartment 2: B-SLIMresolved spectrum

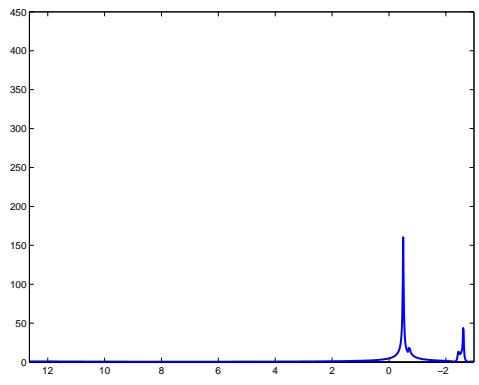

(c) Compartment 1: SLIMresolved spectrum

Figure 3. Synthetic data: algorithm results 


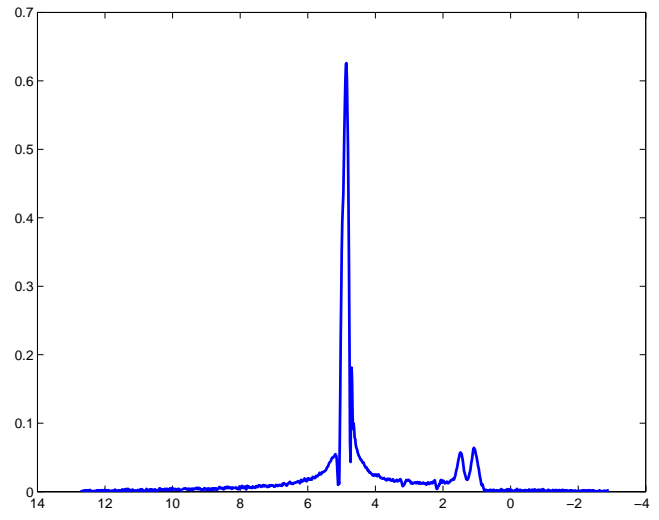

(a) B-SLIM spectrum for compartment 1

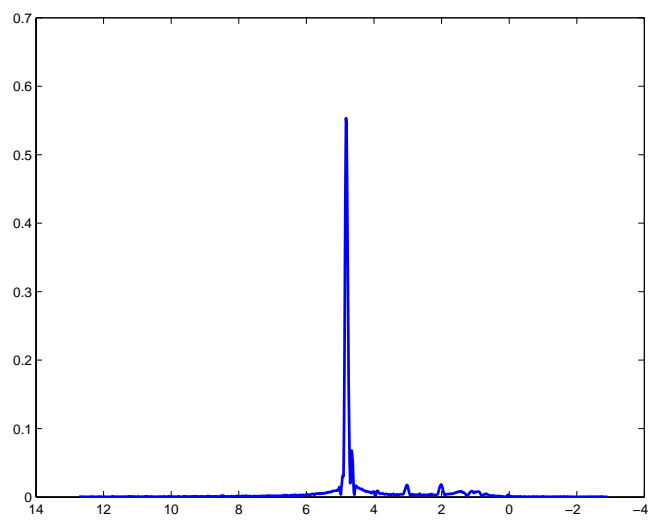

(c) B-SLIM spectrum for compartment 2

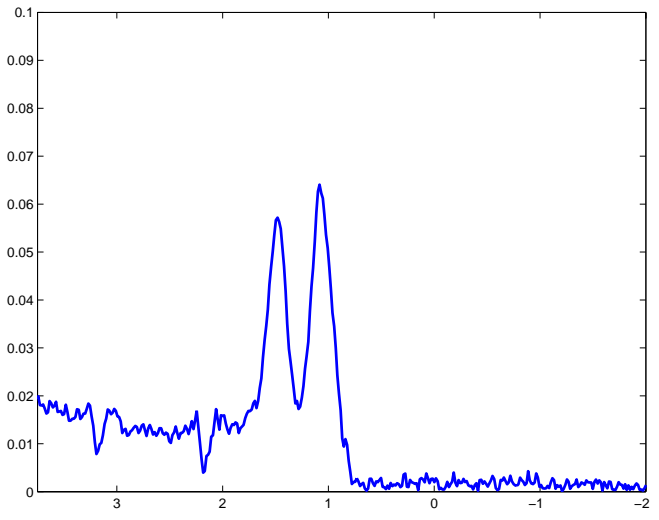

(b) B-SLIM spectrum for compartment 1 (magnified)

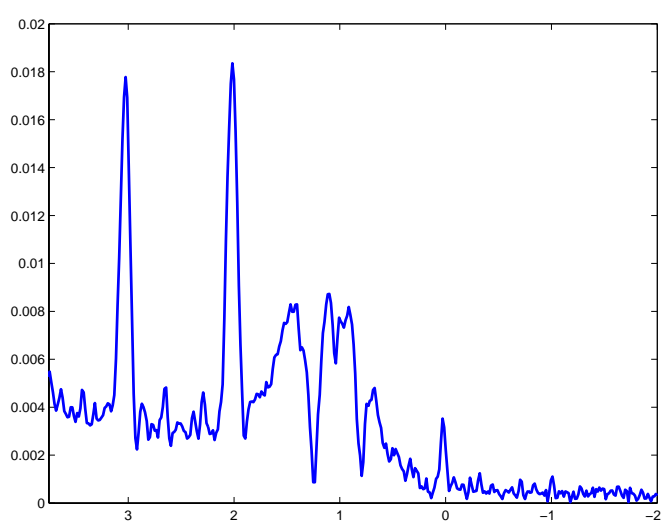

(d) B-SLIM spectrum for compartment 2 (magnified)

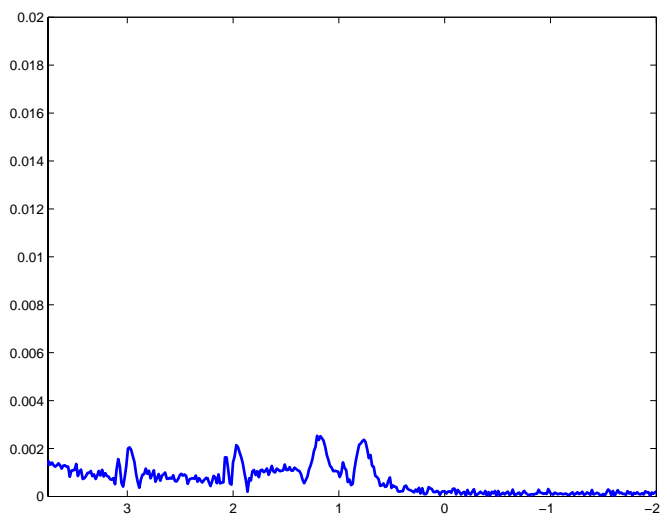

(e) Standard SLIM spectrum for compartment 2 (magnified)

Figure 4. Phantom experiment: results 
The method was implemented and tested with synthetic and physical phantom data. The results show significant improvement over the SLIM technique in terms of spurious peak suppression, visual quality and detectability of the metabolites. These advantages might open a possibility of wide usage of MRSI in challenging applications that are restricted in measurement time, and yet require precise information on the presence of metabolites in the voxels of interest, such as in-vivo investigations and clinical diagnosis.

\section{Acknowledgment}

This work was supported by the Center for Biomedical Imaging (CIBM) of the Geneva - Lausanne Universities and the EPFL, the foundations Leenaards and Louis-Jeantet, as well as by the Swiss National Science Foundation under grant 200020-109415.

\section{REFERENCES}

1. J. Tsao, "Extension of finite-support extrapolation using the generalized series model for MR spectroscopic imaging," IEEE Trans. Med. Imag. 20(11), pp. 1178-1182, 2001.

2. C.-M. Tsai and D. Nishimura, "Reduced aliasing artifacts using variable-density k-space sampling trajectories," Magn. Res. Med. 43(3), pp. 452-458, 2000.

3. R. Gruetter, "Automatic, localized in vivo adjustment of all first- and second-order shim coils," Magn. Res. Med. 29(6), pp. 804-811, 1993.

4. S. Nelson, D. Vigneron, J. Star-Lack, and J. Kurhanewicz, "High spatial resolution and speed in MRSI," NMR in Biomedicine 10(8), pp. 411-422, 1997.

5. L. Yao, Y. Cao, and D. Levin, "2D locally focused MRI: Applications to dynamic and spectroscopic imaging," Magn. Res. Med. 36(6), pp. 834-846, 1996.

6. X. Hu, D. Levin, P. Lauterbur, and T. Spraggins, "SLIM: Spectral localization by imaging," Magn. Res. Med. 8, pp. 314-322, 1988.

7. E. Haacke, Z.-P. Liang, and S. Izen, "Constrained reconstruction: A superresolution, optimal signal-to-noise alternative to the Fourier transform in magnetic resonance imaging," Med. Phys. 16(3), pp. 388-397, 1989.

8. E. Schneider and G. Glover, "Rapid in vivo proton shimming," Magn. Res. Med. 18, pp. 335-347, April 1991.

9. I. Khalidov, D. Van De Ville, M. Jacob, F. Lazeyras, and M. Unser, "B-SLIM: Constrained reconstruction with field inhomogeneity compensation for MRSI," in preparation. 